\title{
Nuevo método para predecir el diámetro mesiodistal de las coronas de caninos y premolares no erupcionados. Estudio Piloto
}

\section{New Method to Predict Mesiodistal Diameter in Unerupted Canine and Premolars Crown. Pilot Study}

\begin{abstract}
Alejandro Lara ${ }^{*}$, Pablo Navarro ${ }^{2}$, Catalina Sandoval ${ }^{3}$, Paulo Sandoval $^{4}$
1: Alumno programa de Magister en Odontología, Universidad de La Frontera, Temuco, Chile. 2:Bioestadístico del Centro de Investigación en Ciencias Odontológicas, Facultad de Odontología Universidad de La Frontera, Temuco, Chile. 3:Fonoaudióloga, Unidad Ortodoncia Interceptiva, Universidad de La Frontera, Temuco, Chile. 4: Ortodoncista Departamento Odontopediatría y Ortodoncia, Universidad de La Frontera, Temuco, Chile.

* Correspondencia autor. Paulo Sandoval Vidal | Avenida Claro Solar 112 cuarto piso, Facultad de Odontología, Temuco, Chile. | Teléfono: +56 452325775 | E-mail: paulo.sandoval@ufrontera.cl Trabajo recibido el 03/10/2018.

Aprobado para su publicación el 27/10/2018

\section{RESUMEN}

La determinación de la discrepancia de la longitud del arco y del tamaño del diente en la dentición mixta, requiere una predicción precisa del ancho mesiodistal de los dientes permanentes no erupcionados, para prevenir futuras maloclusiones. Objetivo: fue generar una nueva ecuación regresiva para predecir el diámetro mesiodistal de premolares y caninos, usando como predictor incisivos inferiores y molares superiores. Material y método: El estudio se realizó en el Centro Clínico de la Araucanía. La muestra está compuesta por modelos dentales históricos de 200 escolares (93 niños y 107 niñas) de toda la IX región, Chile. Edad de 13-16 años. Las mediciones del ancho mesiodistal de incisivos mandibulares, caninos y premolares maxilares y mandibulares, se realizaron con la ayuda del calibrador Vernier calibrado a 0,01 de un milímetro. Resultados: Estos muestran que hay una correlación moderada entre el molar superior y premolares superiores e inferiores. Además, los incisivos inferiores se correlacionan en mayor medida con los caninos superiores e inferiores. No hay diferencias significativas entre hombres y mujeres. Conclusión: Se desarrolló una nueva fórmula con un $75 \%$ de precisión para predecir el ancho de dientes caninos y premolares en dentición mixta en la población chilena de la región de la Araucanía, siendo mejor que la actual fórmula de Tanaka.
\end{abstract}

PALABRAS CLAVE:

Técnicas de apoyo para la decisión; Dentición mixta; diente; discrepancia; Ortodoncia interceptiva; Molar.

Rev. Clin. Periodoncia Implantol. Rehabil. Oral Vol. 12(1); 19-22, 2019

\section{ABSTRACT}

Precautionary measures are necessary to prevent a potential irregularity from progressing into a more severe malocclusion. The determination of the tooth size-arch length discrepancy in mixed dentition requires an accurate prediction of the mesiodistal widths of the unerupted permanent teeth to prevent future malocclusions. Objective: The purpose of this study is to generate a new regressive equation to predict the diameter of the cuspid and bicuspid by measuring the wide of lower incisor and upper molar teeth in a Chilean population sample. Matherials and methods: the study was conducted in the Araucanía Clinical Center in Temuco city and the sample was composed of historical dental cast from 200 school children (93 boys and 107 girls), of IX region, Chile. Age group of 13-16 years old. Measurements of the mesiodistal widths of mandibular incisors, maxillary and mandibular canines and premolars were made with the help of Vernier gauge calipers calibrated to 0.01 of 1 millimeter. Results: The results show that there is a moderate correlation between the upper molar and upper and lower premolars. In addition, the lower incisors are correlated to a greater extent with the upper and lower canines. There are no significant differences between men and women. Conclusion: A new formula with $75 \%$ accuracy was developed to predict the width of canine teeth and premolars in mixed dentition in the Chilean population of the Araucanía region. This formula is better than the current Tanaka method.

KEY WORDS:

Techniques of support for the decision; Mixed dentition; Tooth size; Discrepancy; Interceptive orthodontics; Molar; Predictive formula.

Rev. Clin. Periodoncia Implantol. Rehabil. Oral Vol. 12(1); 19-22, 2019 


\section{INTRODUCCIÓN}

La maloclusión dental es una condición muy común en las poblaciones de todo el mundo. La discrepancia entre la longitud del arco y el tamaño de los dientes se considera un factor etiológico universalmente importante. Si el tamaño del diente y la dimensión del arco se estiman con precisión antes del establecimiento de la maloclusión, es posible realizar procedimientos preventivos ${ }^{(1)}$. Es por lo anterior que en la planificación del tratamiento de ortodoncia se utilizan los métodos radiográficos(2), modelos dentales ${ }^{(3)}$, y una combinación de métodos radiográficos y dentales ${ }^{(4)}$.

Los principales problemas de ortodoncia observados en la fase de dentición mixta, están relacionados con la longitud del arco y la discrepancia con el tamaño del diente. Con el fin de identificar tales discrepancias de espacio, se han introducido diferentes métodos de análisis en dentición mixta. El propósito de estos métodos, es evaluar la cantidad de espacio disponible en el arco para los futuros dientes permanentes y el ajuste oclusal(5). Se han publicado diferentes métodos para predecir el tamaño de los caninos y permanentes ${ }^{(6)}$. Los primeros intentos de estimar el tamaño de los dientes fueron los realizados por Black ${ }^{(7)}$. Otros métodos de predicción se basaron en la estimación del tamaño de los dientes permanentes en radiografías por sí solos o en combinación con diámetros de corona, medidos en moldes dentales ${ }^{(8)}$. Un método ampliamente utilizado correlaciona los diámetros de la corona mesiodistal de los incisivos permanentes mandibulares erupcionados, como el predictor del tamaño de los caninos y premolares no erupcionados ${ }^{(4)}$. Entre los diferentes métodos disponibles, el análisis de dentición mixta de Tanaka y Johnston es probablemente el más utilizado $^{(9)}$. Sus tablas de probabilidad se desarrollaron a partir de estudios realizados en población caucásica ${ }^{(10)}$. Este es un método de correlación que usa el ancho mesiodistal de los incisivos permanentes mandibulares erupcionados para predecir el ancho mesiodistal de los caninos y premolares no erupcionados, con el uso de una tabla de predicción. Sin embargo, se ha estudiado la aplicabilidad de estas tablas en otros grupos étnicos obteniéndose resultados deficientes ${ }^{(12,13)}$ Además, en la literatura está bien establecido que el ancho mesiodistal de los dientes varía considerablemente entre los diferentes grupos raciales ${ }^{(14,15)}$. El dismorfismo sexual también se ha confirmado en varios estudios $^{(14)}$. Como una manera de estudiar las variaciones de tamaño dentario endentición mixta, se han realizado múltiples estudios en que se proponen nuevas formas para mejorar las predicciones y obtener resultados más precisos ${ }^{(16-18)}$.

El objetivo del presente estudio, es generar una nueva ecuación de predicción para la estimación del ancho mesiodistal de los caninos y premolares maxilares, basados en el ancho de los incisivos inferiores y de los primeros molares permanentes superiores.

\section{MATERIALES Y MÉTODOS}

El estudio se realizó en el Centro de Ortodoncia Araucanía de la ciudad de Temuco (marzo - agosto 2014) y la muestra fue compuesta por una colección histórica de modelos dentales de 200 escolares (93 niños y 107 niñas), los cuales fueron derivados de centros de atención odontológica, públicos y privados de toda la IX Región (de la Araucanía), de edades entre 13-16 años, (95 hombres y 105 mujeres). Las indicaciones de ingreso de los pacientes consisten en la presencia de anomalías dentomaxilares de baja mediana y alta complejidad. Cabe destacar, que el universo de pacientes es representativo de la Novena Región en el periodo de estudio, el segundo centro referente de la especialidad en la zona. Se realizaron análisis de regresión lineal entre los grupos dentarios y se desarrolló un nuevo índice predictivo.

\section{Criterios de selección}

1. Los modelos dentales fueron obtenidos de niños en el grupo de edades entre 13-16 años. 2. Todos los dientes permanentes debían estar presentes en cada arco dental y debiendo estar erupcionados por completo (con excepción de terceros molares) 3. Los dientes permanentes tenían que estar libres de caries. 4. Se excluyeron los niños con anomalías craneofaciales congénitas o antecedentes de ortodoncia previa. 5. Los niños con restauraciones o atrición significativa fueron excluidos.

2. Las impresiones de los arcos maxilares y mandibulares fueron realizadas con alginato como material de impresión de los niños seleccionados. Los modelos de estudio se prepararon cuidadosamente utilizando yeso piedra dental. Se usó un método estandarizado para medir el ancho de la corona mesiodistal ${ }^{(19)}$. El ancho de la corona mesiodistal más grande de cada diente se midió entre sus puntos de contacto, con las pinzas del calibrador Vernier calibrados a 0,01 de un milímetro (Uyustools, China), colocándose paralelos a las superficies oclusales y vestibulares. En la literatura se ha reportado que este método es altamente preciso para medir el ancho de la corona mesiodistal ${ }^{(20)}$. La fiabilidad de la medición se verificó según el método sugerido por Lundstorm, donde un único investigador realiza todas las mediciones después de marcar cuidadosamente el ancho mesiodistal máximo en los dientes a medir y luego se vuelven a medir ciertos moldes seleccionados al azar ${ }^{(21)}$. Se calculó el coeficiente de fiabilidad de la prueba en 20 de dichos moldes seleccionados al azar. El valor $\mathrm{R}$ fue de 0,97 , por lo que se confirmó la fiabilidad.

La suma de las medidas mesiodistal de los siguientes grupos de dientes se catalogaron y se calcularon los promedios: -los cuatro incisivos inferiores- los caninos y premolares mandibulares, los caninos maxilares y los premolares en cada cuadrante, y los primeros molares superiores e inferiores. Estos datos luego se usaron para la ecuación de regresión entre diferentes grupos de dientes, que podría usarse clínicamente para predecir los tamaños de los dientes correlacionando los grupos anteriores entre sí, es decir, los incisivos con los caninos y los dientes posteriores entre sí, es decir, los molares con los premolares, determinando los mejores predictores.

\section{Análisis estadístico}

Los datos recopilados se sometieron a un análisis de regresión para correlacionar los modelos para los diferentes grupos de dientes, utilizando SPSS IBM Statistics para Windows versión 23.0 (Chicago, IL.).

\section{RESULTADOS}

La muestra ha sido categorizada en subgrupos por el tamaño mesiodistal de incisivos, caninos y premolares para generar una ecuación lineal regresiva entre cupos.

Las medidas de los anchos mesiodistal de los dientes se resumen en las tablas I y II.

Tabla 1. Tamaño promedio mesiodistal de los incisivos y caninos maxilares y mandibulares.

\begin{tabular}{|l|l|l|l|}
\hline Grupo dentario & Hombres & Mujeres & Totales \\
\hline & Promedio/ DS & Promedio/DS & \\
\hline Incisivo inferior & $11.82 / 0.78$ & $11.62 / 0.78$ & $11.71 / 0.78$ \\
\hline Incisivo superior & $16.13 / 1.05$ & $15.86 / 1.13$ & $15.99 / 1.09$ \\
\hline $\begin{array}{l}\text { Molares } \\
\text { superiores }\end{array}$ & $10.74 / 0.57$ & $10.50 / 0.47$ & $10.62 / 0.53$ \\
\hline $\begin{array}{l}\text { Premolares } \\
\text { inferiores }\end{array}$ & $14.79 / 0.86$ & $14.58 / 0.83$ & $14.68 / 0.85$ \\
\hline $\begin{array}{l}\text { Premolares } \\
\text { superiores }\end{array}$ & $14.26 / 0.93$ & $14.13 / 0.90$ & $14.19 / 0.91$ \\
\hline
\end{tabular}

Tabla 2. Análisis de regresión entre anchos mesiodistales de grupos dentarios anteriores.

\begin{tabular}{|l|l|l|l|l|}
\hline Variables & $\mathrm{P}$ & $\mathrm{R}$ & $\mathrm{F}$ & Sig. \\
\hline Canino superior/ incisivo inferior & $<0.05$ & 0.443 & 48.63 & $0.001^{*}$ \\
\hline Canino superior / incisivo superior & $<0.05$ & 0.520 & 73.570 & $0.001^{*}$ \\
\hline Canino inferior / incisivo inferior & $<0.05$ & 0.634 & 133.476 & $0.001^{*}$ \\
\hline Canino inferior / incisivo superior & $<0.05$ & 0.556 & 89.047 & $0.001^{*}$ \\
\hline
\end{tabular}

*: Diferencias significativas

Tabla 3. Análisis de regresión entre anchos mesiodistales de grupos dentarios posteriores.

\begin{tabular}{|l|l|l|l|l|}
\hline Variables & $\mathrm{P}$ & $\mathrm{R}$ & $\mathrm{F}$ & Sig. \\
\hline Premolares superiores $/ 1^{\circ}$ molar inferior & $>0.05$ & 0.181 & 6.715 & $0.01^{*}$ \\
\hline Premolares superiores $/ 1^{\circ}$ molar superior & $<0.05$ & 0.458 & 52.701 & $0.001^{*}$ \\
\hline Premolares inferiores $/ 1^{\circ}$ molar inferior & $<0.05$ & 0.414 & 41.139 & $0.001^{\star}$ \\
\hline Premolares inferiores $/ 1^{\circ}$ molar superior & $<0.05$ & 0.452 & 51.002 & $0.001^{*}$ \\
\hline
\end{tabular}

*: Diferencias significativas 
Del gráfico 1 se puede inferir que, a medida que aumenta el tamaño mesio distal de 4.1+4.2, aumenta el tamaño de 1.3.El R2(Coeficiente de determinación), nos indica que el $19,6 \%$ de toda la variabilidad, que ocurre en la medida del canino superior se explica por la variabilidad que ocurre en la suma de la medida de los insicivos inferiores.

Del gráfico 2 se puede inferir que, a medida que aumenta el tamaño mesio distal de incisivos inferiores, aumenta el tamaño del canino inferior. El R2 en este caso nos indica que el $40,1 \%$ de toda la variabilidad que ocurre en la medida del canino inferior, se explica por la variabilidad que ocurre en la suma de la medida de los insicivos inferiores.

Del gráfico 3 se puede inferir que, a medida que aumenta el tamaño mesio distal del molar superior, aumenta el tamaño de los premolares superiores. El Coeficiente de determinación (R2) de este gráfico, nos indica que el $20,9 \%$ de toda la variabilidad, que ocurre en la medida de los premolares superiores se explica por la variabilidad que ocurre en la medida del $1^{\circ}$ molar superior.

Del gráfico 4 se puede inferir que, a medida que aumenta el tamaño mesio distal del $1^{\circ}$ molar superior, aumenta el tamaño de los premolares inferiores y el $20,4 \%$ de toda la variabilidad, que ocurre en la medida los premolares inferiores se explica por lo que ocurre en la medida del $1^{\circ}$ molar superior (R2).

Basado en este análisis, se ha establecido un buen modelo regresivo para los incisivos inferiores, como predictor del ancho de los caninos inferiores. Los incisivos superiores fueron un buen predictor para los caninos superiores, sin embargo, las diferencias no son significativas con el inferior.

Se puede observar que la relación entre los incisivos inferiores y el tamaño de los caninos es positiva, lo cual indica que, a mayor tamaño de los incisivos inferiores, mayor es el tamaño de los caninos. Así mismo, la relación entre el molar superior y la suma de los anchos de los premolares, es positiva también.

\section{DISCUSIÓN}

Se determinaron cuatro ecuaciones de regresión lineal para predecir los caninos y los premolares en los dientes maxilares. Se establecieron otras dos ecuaciones para los dientes mandibulares. Utilizando el primer molar maxilar y dos incisivos mandibulares de forma independiente como predictores. Las ecuaciones así determinadas se calcularon como $Y=a$ $+\mathrm{bx}$, donde $\mathrm{Y}$ es la variable dependiente y $\mathrm{X}$ es la variable independiente (Ver gráficos 1 al 4 ).

\section{Dispersión entre 4,1 y 4,2 v/s 1,3}

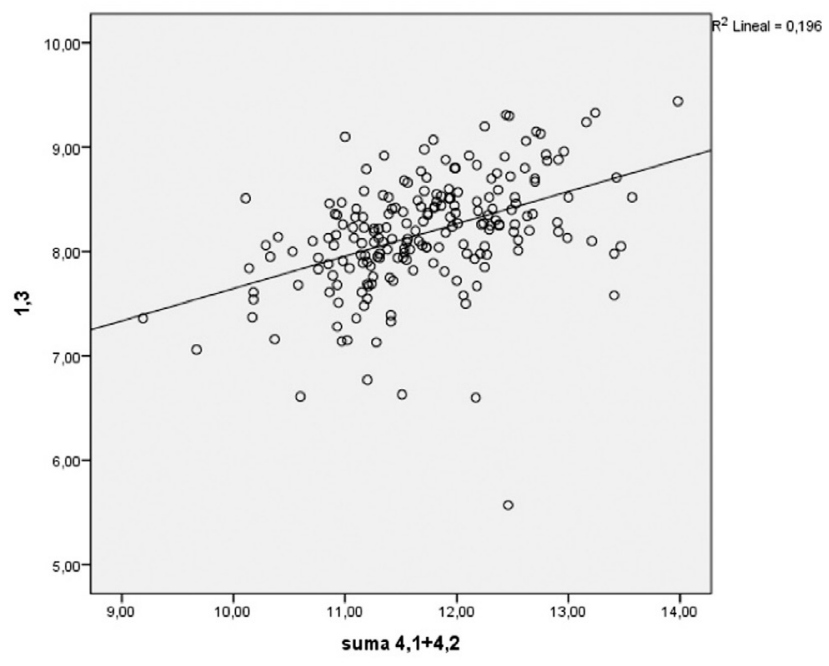

\section{Ecuación de regresión $Y=4,546+0.310 x$}

Gráfico 1. Diagrama de dispersión entre sumatoria de incisivos inferiores y caninos maxilares en una muestra de 200 sujetos de la región de La Araucanía.

Con respecto a los datos sobre el tamaño de los dientes de la muestra, el valor del coeficiente de predicción es confiable, preciso y por tanto útil clínicamente. El tamaño del incisivo inferior se puede utilizar para predecir el ancho del canino y el tamaño del molar para predecir el ancho de los premolares.

\section{Dispersión entre 4,1 + 4,2 v/s 4,3}

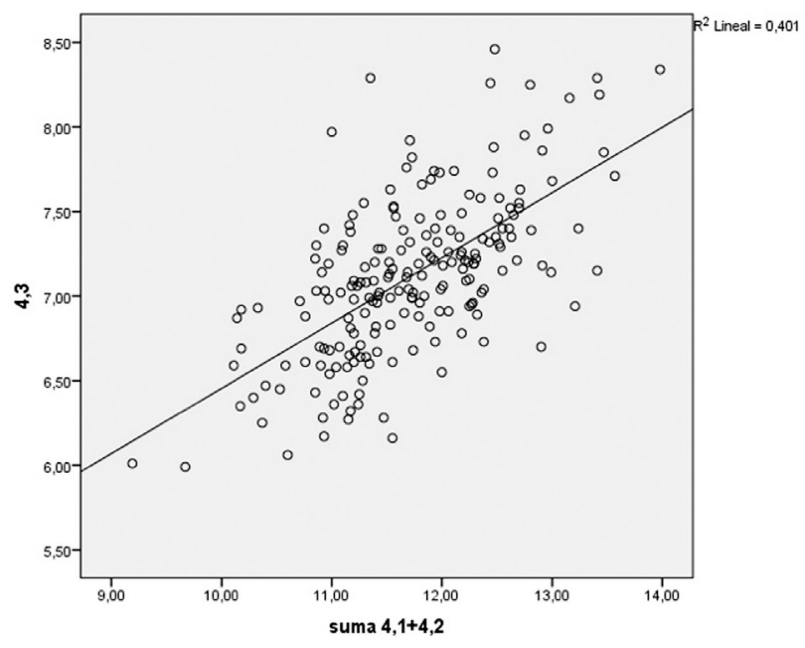

Ecuación de regresión $Y=2,598+0.386 x$

Gráfico 2. Diagrama de dispersión entre sumatoria de incisivos inferiores y caninos mandibulares en una muestra de 200 sujetos de la región de La Araucanía.

\section{Dispersión entre1,6 v/s SUMA 1,4 + 1,5}

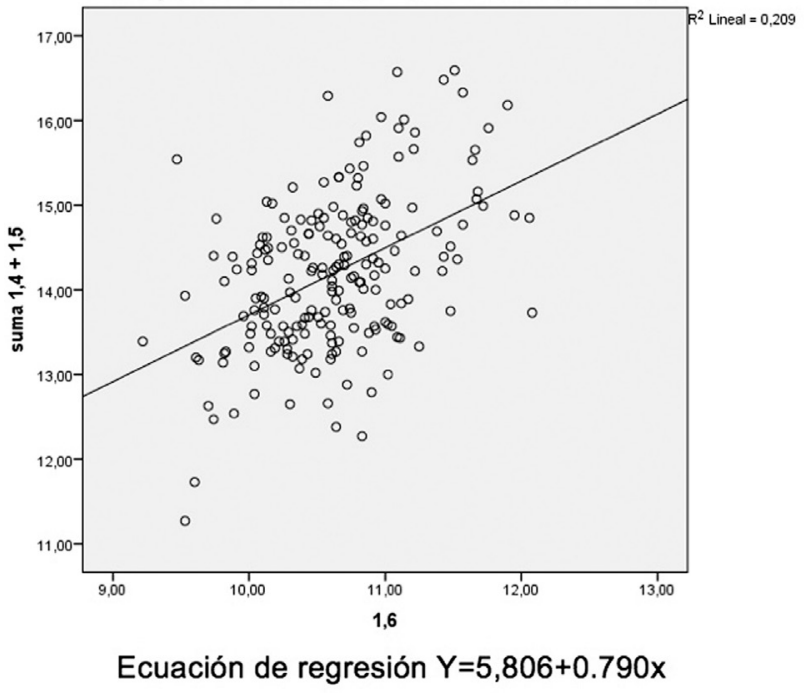

Gráfico 3. Diagrama de dispersión entre molar maxilar y sumatoria de premolares superiores en una muestra de 200 sujetos de la región de La Araucanía.

Este estudio se realiza con el fin de buscar una mejor predicción de tamaños dentarios en dentición mixta, toda vez que la predicción de Tanaka es muy baja de acuerdo a diferentes autores ${ }^{(22-23)}$. Así como para generar una nueva fórmula simplificada de aplicación clínica para la población local. Con el fin de garantizar la fiabilidad de los datos, se tomó un tamaño de muestra suficientemente grande $(n=200)$, con una edad comprendida entre 13 y 16 años.

Hay múltiples ecuaciones predictivas propuestas realizadas, pero todas ellas coinciden en que los valores se deben ajustar a cada población o región ${ }^{(24)}$

Melgaco et al ${ }^{(25)}$, incorporan el molar a una fórmula general similar a la tradicional ecuación de Tanaka ${ }^{(9)}$, sin embargo utilizaron esta variable mezclada con el tamaño de los incisivos junto a los molares para obtener una sola nueva fórmula. Del mismo modo Shahid et al ${ }^{(18)}$,también proponen utilizar el molar inferior como variable predictiva en conjunto con los incisivos.

No se encontraron reportes en que se haya establecido correlaciones de forma independiente entre grupos posteriores y anteriores, siendo 


\section{Dispersión entre1,6 v/s SUMA 4,4 + 4,5}

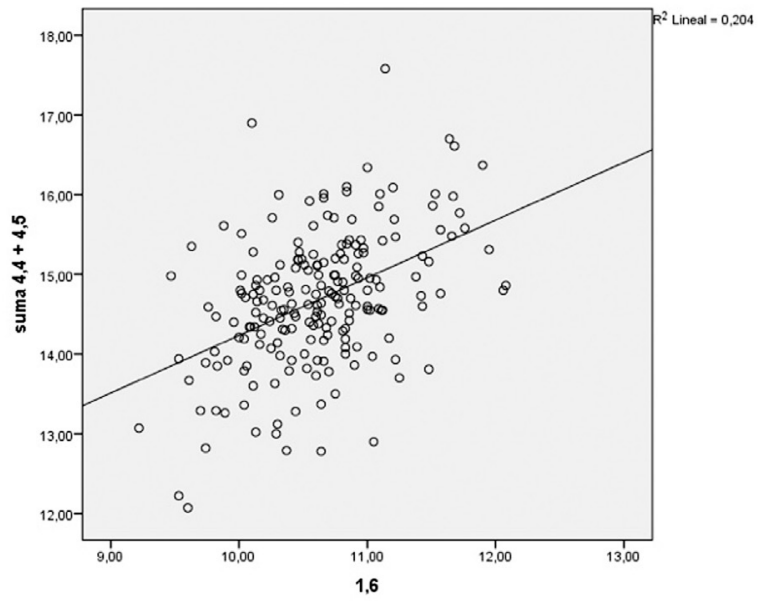

Ecuación de regresión $Y=7,002+0.723 x$

Gráfico 4. Diagrama de dispersión entre molar maxilar y sumatoria de premolares inferiores en una muestra de 200 sujetos de la región de La Araucanía. esta investigación la primera en explorar esta alternativa.

Teniendo los grupos que mejor se correlacionan como predictores, se presentan las siguientes fórmulas simplificadas en base a los promedios de tamaños dentarios, para calcular clínicamente la predicción:

En la maxila: [Suma de incisivos inferiores $/ 2-3,5 \mathrm{~mm}]+[$ molar superior $(1.6)+3,6 \mathrm{~mm}]=$ Diente 1.3 o $2.3+$ premolares superiores $(1.4+1.5)$

En la Mandíbula: [Suma de incisivos inferiores/2 - 4,6mm]+ [molar superior $(1.6)+4,0 \mathrm{~mm}]=$ Diente 3.3 o $4.3+$ premolares inferiores $(4.4$ $+4.5)$

Con esta fórmula, en la maxila hubo $50 \%$ de los pacientes con error menor de 0,5 milímetros, $30 \%$ de los individuos entre 0,5 y $1 \mathrm{~mm}$ y $20 \%$ mayor a $1 \mathrm{~mm}$.

Debido a la ausencia de literatura con similar enfoque en la formulación de esta ecuación de regresión para nuestra población, el presente estudio intenta desarrollar una fórmula clínica simplificada para con niños de Temuco.

\section{CONCLUSIÓN}

A pesar de que existe disformismo sexual en el tamaño de los dientes, este no es significativo. En consideración a que la ecuación de regresión lineal fue desarrollada con datos obtenidos de una muestra representativa de la población de la novena región, esta es más precisa que las fórmulas internacionales usadas actualmente (Métodos de Tanaka y Moyers), obteniendo un $75 \%$ de precisión para aplicaciones clínicas. Es necesario validar esta fórmula, en una muestra representativa de la población de cada región o del país para ser utilizada como estándar regional o nacional.

\section{CONFLICTO DE INTERESES}

Los autores declaran no tener conflicto de interés.

\section{Bibliografía}

1. Sim JM. Minor tooth movements in children. 2nd ed. St Louis: Mosby; 1977. 2. Smith HP, King DL, Valencia RA. Comparison of three methods of mixed dentition analysis. J Pedod. 1979; 3(4): 291-302.

3. Staley RN, O'Gorman TW, Hoag F, Shelly TM. Prediction of the width of unerrupted canines and premolars. J Am Dent Assoc. 1984; 108(2): 185-190.

4. Motokawa W, Dzakim SY, Yoshida YA. Method of mixed dentition analysis in the mandible. J Dent Child. 1987; 54(2): 114-118.

5. Diagne F, Diop-Ba K, Ngom PI, Mbow K. Mixed dentition analysis in Senegalese population and elaboration of prediction tables. Am J Orthod Dentofacial Orthop. 2003; 124(2): 78-83.

6. Yuen KK, Tang EL, So LL. Mixed dentition analysis for Hong Kong Chinese. Angle orthod. 1998; 68(1): 21-28.

7. Black GV. Descriptive anatomy of human teeth. 4th ed. Philadelphia: S.S. White Dental Manufacturing; 1897.

8. Gardner RB.A comparison of four methods of predicting arch length. Am J Orthod. 1978;75:387-97.

9. Tanaka MM, Johnston LE. Prediction of the sizes of unerrupted canines and premolars in a contemporary orthodontic population. J Am Dent Assoc. 1974; 88(4): 198-801.

10. Ballard ML, Wylie WL. Mixed dentition case analysis: Estimating size of unerrupted permanent teeth. Am J Orthod. 1947; 33(11): 754-759.

11. Staley RN, Kerber PE. Revision of Hixson and Oldfather mixed dentition prediction method. Am J Orthod. 1980; 78(3): 296-302.

12. Frankle $\mathrm{HH}$, Benz EM. Mixed dentition analysis for Black Americans. Pediat Dentistry. 1986; 8(3): 226-230.

13. Ferguson FS, Macko DJ, Sonnenberg EM, Shakn ML. The use of regression constants in estimating tooth size in Negro population. Am J Orthod. 1978; 73(1): 68-72.

14. Shirmer UR, Wiltshire WA. Orthodontic probability tables for Black patients of African descent: Mixed dentition analysis. Am J Orthod Dentofacial Orthop. 1997; 112(5): 545-551.
15. Bailit HL. Dental variation among population. Dent Clin North Am. 1975; 19(1): 125-139.

16. Martinelli FL, de Lima EM, Rocha R, Tirre-Araujo MS. Prediction of lower permanent canine and premolars width by correlation methods. Angle Orthod 2005; 75(5): 805-808.

17. Nourallah AW, Gesch D, Khordaji MN, Splieth C. New regression equations for predicting the size of unerupted canines and premolars in a contemporary population. Angle Orthod. 2002; 72(3): 216-221.

18. Shahid F, Alam MK, Khamis MF. New predict equations for the estimation of maxillary mandibular canines and premolars widths from mandibular incisors and mandibular first permanent molar width: A digital model study. Korean J Orthod 2016;46(3):171-179.

19. Moorrees CF, Reed RB. Correlations among crown diameters of human teeth. Arch Oral Biol. 1964; 9(6): 685-697.

20. Doris JM, Bernard BW, Kuftinec MM, Stom DD. A biometric study of tooth size and dental crowding. Am J Orthod. 1981; 79(3): 326-336.

21. Hixon EH, Oldfather RE. Estimation of the sizes of unerruptedcuspid and bicuspid teeth. Angle Orthod. 1958; 28(4): 236-240.

22. Jaroontham RJ, Godfrey KR. Mixed dentition space analysis in a Thai population. Eur J Orthod. 2000; 22(2): 127-134.

23. Philip IN, Prabhakar M, Arora D, Chopra S. Applicability of the Tanakas mixed dentition probability tables and new prediction aids for a contemporary population in India. Am J Orthod Dentofacial Orthop. 2010; 138(3): 339-345.

24. Bernabe E, Flores-Mir C. Are the lower incisors the best predictions for the unerupted canine and premolars sums? An analysis of a Peruvian sample. Angle Orthod. 2005; 75(2): 198-203.

25. Melgaco AC, Sousa Araujo MT. Mandibular permanent first molar and incisor width as predictor of mandibular canine and premolar width. Am J Orthod Dentofacial Orthop. 2007; 132(3): 340-345. 